\title{
Experimental-numerical method for evaluation of energy consumption in percussive drilling of granite
}

\author{
Wei Yao*, and Mikko Hokka \\ Impact - Multiscale Mechanics Research Group, Engineering Materials Science, Tampere University, \\ POB 589, FI-33014, Tampere, Finland
}

\begin{abstract}
Most geothermal energy is found in hot and dry rock (HDR), and the total resources distributed at depths of 3-10 km are $20.9 \times 10^{6}$ EJ. Most HDR projects are developed in granite formations and understanding and control of the energy consumption in percussive drilling of granite is essential. Thus, the impact energy is dissipated in various processes including fracture of the granite, kinetic energy of the particles, and heat. This study presents a numerical-experimental method to evaluate the energy consumption in percussive drilling process. The method uses particle flow code (PFC2D) and experiments using a modified Hopkinson pressure bar system. Optical high-speed and infrared cameras were used to record the impact process during the dynamic drilling process. The velocity of fragments was estimated by the high-speed images and the instantaneous temperature change during the impact was observed from infrared images. Preliminary results are given in this study indicating that the proposed numerical and experimental methodologies can be used to investigate the energy consumption during percussive drilling. The preliminary results presented in this paper are part of a larger work in progress, and more work needs be carried out to obtain the energy consumption of the drilling process under different loading and boundary conditions.
\end{abstract}

\section{Introduction}

Geothermal energy, as a pollution-free and renewable energy resource, exists in rock masses below the Earth's Surface [1]. Most geothermal energy is found in hard and dry rock (HDR), and the total resources distributed at depths of $3-10 \mathrm{~km}$ are $20.9 \times 10^{6} \mathrm{EJ}$ [2]. Most HDR projects are developed in granite formations [3], and the energy consumption of percussive drilling of HDR is different from that of other rocks. With the prosperity of the experimental and computing techniques, many theoretical, numerical and experimental advances in laboratories have been highly utilized for the assessment and improvement of energy efficiency of drilling. This work on improving energy efficiency and performance of drilling aims at reduced costs and improved reliability [4-6]. However, complexity of the integrated process of rock drilling involving many disciplines and complicated physics leads to

* Corresponding author: yaoweiut@hotmail.com 
significant theoretical challenges in the rigorous modelling of the full drilling process. Therefore, the recent advances in improved drilling energy efficiency have been rather empirical and driven by qualitative knowledge rather than by comprehensive and quantitative insights of the drilling process. Consequently, so far both the energy efficiency and understanding of the energy efficiency of drilling remains poor.

Most of the energy used for drilling is not consumed in fragmenting the rock, but instead, the majority of the energy is spent in heating, kinetic energy, tool wear, etc. Furthermore, the specific energy, i.e. the work done to excavate a unit volume of rock, has been widely and continuously used to assess the energy efficiency of percussive drilling in real-time [7]. The specific energy, however, represents insufficiently the efficiency of the rock fragmentation during the drilling process of dry hard rock. Also, the specific energy alone does not ensure that the drilling crew knows what the root cause of the observed inefficiency of the drilling is or how to correct it. Therefore, recent studies have focused on the factors that reduce the efficiency and prohibit the energy from being properly transferred to the formation, causing a large portion of the input energy to go to waste [8]. The results have indicated that the dynamic tool-rock interaction processes and rock-breaking mechanisms have to be considered in the analysis of the energy efficiency of percussive drilling [9]. However, the research has yet to quantify the energy consumption of drilling and to integrate dynamic tool - hard rock interaction processes with the distribution of energy consumption to different processes, e.g., heating, kinetic energy, and fracture energy. In this study, a conceptual study was conducted to investigate energy consumption in drilling of granite for deep geothermal exploitation. The numerical and experimental methods proposed in this work allow systematic evaluation of the energy consumption in different processes, e.g., heating, kinetic energy of particles etc., in percussion drilling of granite. This paper presents preliminary results of work in progress.

\section{Numerical modelling of dynamic drilling process for evaluation of energy consumption during drilling}

Numerical models in two-dimensional (2D) particle flow code (PFC) are composed of discrete circular particle aggregates, in which the particles are considered as rigid and the contacts among the particles are restricted to a small area. The movements of the particles follow the Newtonian second law and the contact force is obtained by using the inherent relationship between force and displacement. Since an explicit method is used in the PFC to solve the equations of motion, it can be efficiently applied to conduct the dynamic simulation.

The numerical dynamic indentation model was constructed to mimic the dynamic percussive drilling process, as shown in Fig. 1. The length of the bar is $1 \mathrm{~m}$ and the diameter of the bar is $20 \mathrm{~mm}$. The diameter of the drill bit is $5 \mathrm{~mm}$. In the simulation, the radius range of particles in the incident bar is between 0.55 and $1.1 \mathrm{~mm}$ and those in the single drill bit is between 0.05 and $0.125 \mathrm{~mm}$. The density of particles is $7800 \mathrm{~kg} / \mathrm{m}^{3}$ deduced from the mass conservation law. The microscopic deformation parameters of particles for the bar and the drill bit are $5.43 \times 10^{11}$ for the normal stiffness and $6.87 \times 10^{11}$ for the shear stiffness. These are obtained via a static numerical compressive test based on the real physical parameters of the bar and drill bit materials. As the bar and the drill bit should barely be damaged during the whole impact test, the contact bond strength of these materials is assumed to be high enough (i.e. $1 \times 10^{99} \mathrm{MPa}$ ) to avoid permanent damage. The rock block was constructed by practices with the radius of $0.5-1 \mathrm{~mm}$. The granite properties used in the rock block are shown in Fig. 1 , including the porosity of 0.002 , the normal stiffness of $82 \times 10^{9} \mathrm{~N} / \mathrm{m}$, the shear stiffness of $45 \times 10^{9} \mathrm{~N} / \mathrm{m}$, the particle density of $2500 \mathrm{~kg} / \mathrm{m}^{3}$. The size of the rock block is $100 \mathrm{~mm} \times 100 \mathrm{~mm}$. 


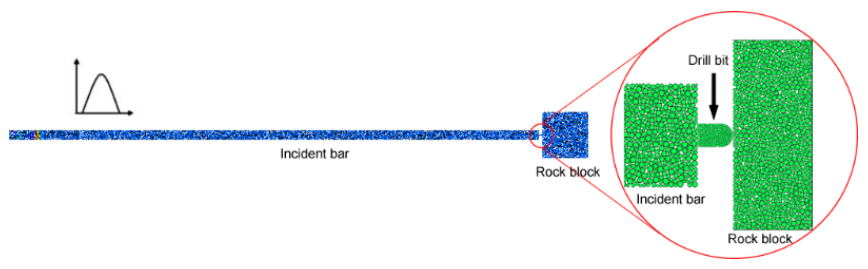

Fig. 1. Numerical dynamic indentation model in 2D PFC.

A stress pulse was applied to the particle group of the incident bar to simulate the impact of the striker on the free end of the incident bar. To inspect the validity of the numerical dynamic experiment, the stress wave propagation in the incident bar was evaluated as shown in Fig. 2. The sequence of images indicates that the stress wave propagates smoothly forth and back in the incident bar and interacts with the rock block. Fig. 3 shows the incident and reflected pulses in the incident bar. Therefore, this numerical model can be used to efficiently simulate the dynamic impact process.

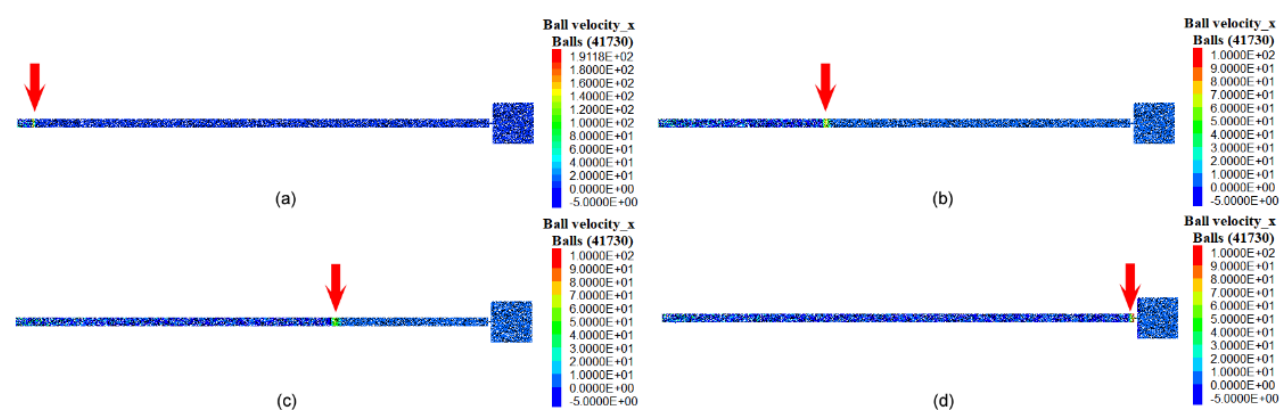

Fig. 2. The stress pulse propagation from the left to right in the incident bar. The location of the stress pulse is annotated by the arrow in each figure and the interframe time is $24.8 \mu \mathrm{s}$.

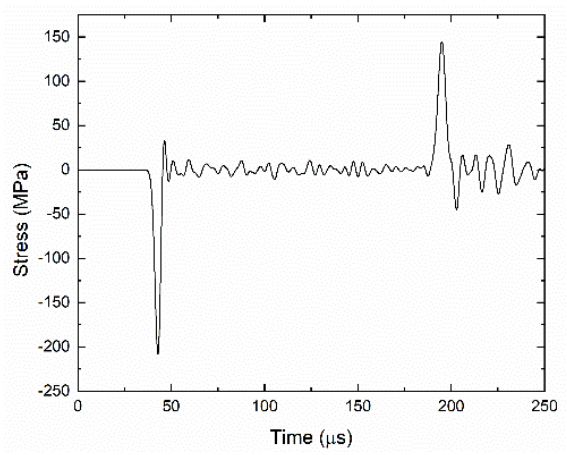

Fig. 3. The incident and reflected stress pulses in the incident bar.

Fig. 4 shows the fragmentation process in the simulation, where the rock was damaged by the impact of the drill bit button. The cracks and the fragments can be produced by this simulation. It can be seen that first cracks were created and then later also the fragments. With the movement of the drill bit, the cracks continue to extend, and the fragments fly out of the rock block with the high speed. The crack lengths can be obtained and the fracture energy during the dynamic indentation can be analysed using this simulation model. Also, the velocities of the fragments can be detected by the 2D PFC and then analysed to get the kinetic energy of the fragments. Therefore, this model can be used to study the energy consumption during the dynamic percussive drilling and more future work can be conducted to investigate the details of the energy consumption in the percussive drilling process. 


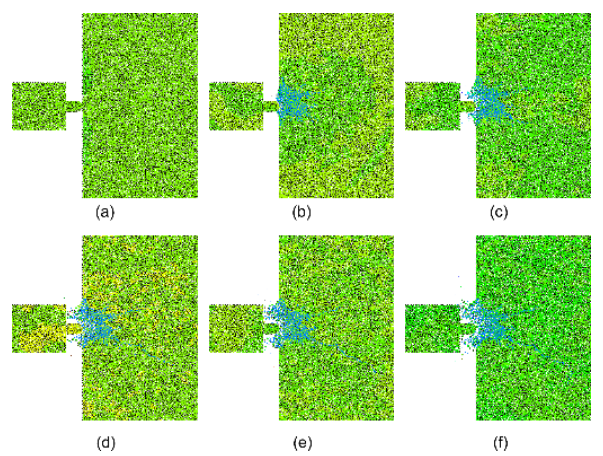

Fig. 4. The fragmentation in the dynamic indentation process (the interframe time is $50 \mu \mathrm{s}$ ).

\section{Experimental method for evaluating energy consumption in percussive drilling}

The energy consumption in the dynamic percussive drilling system was evaluated using a modified split Hopkinson pressure bar (SHPB) system which is shown in Fig. 5. Three drill buttons were mounted at the end of the incident bar to mimic a drill bit. The striker (which can be considered as a hammer) was launched by the air gun to impact the free end of the incident bar. The temperature change of the interface between the drill bit and the rock at the impact moment was estimated by using the high-speed infrared camera placed perpendicular to the impact face. Also, an optical high-speed camera was used to image the fragmentation process during the dynamic impact. A plexiglass cover was used to collect the fragments after the impact. The strain gauges on the incident bar were employed to measure the force history during the dynamic process.

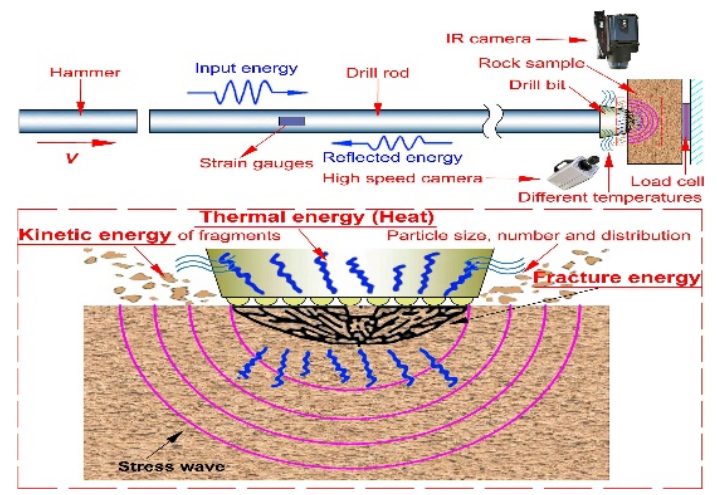

Fig. 5. The schematic picture of the experiment used to evaluate the energy consumption during percussive drilling.

The dynamic force of a typical test is shown in Fig. 6a, whereas the craters and the fragments after a typical test are shown in Figs. $6 \mathrm{~b}$ and c. The preliminary results indicate that the dynamic experimental system can reproduce and experimentally simulate a dynamic percussive process in a laboratory scale. Moreover, the movement of the fragments were captured by using the high-speed camera, as shown in Fig. 7. The velocities of the fragments can be estimated using the series of the high-speed images. At the same time, the radiometric temperature change at the contact between the button and the rock can be detected by the infrared images as shown in Fig. 8. The radiometric temperature in Fig. 8a is approximately $21.2^{\circ} \mathrm{C}$ and then during the impact it increases to $22.9^{\circ} \mathrm{C}$ (in Fig. 8 b). It can be seen that the 
changes in the radiometric temperature are very small before and after the impact. The temperature of the ejecting particles is slightly higher than the original temperature. The temperature changes at the contact can also contribute to the thermal energy losses during the drilling process. However, it is very clear that the temperature changes are very small and that their contribution to the total energy consumption are most likely very small.

As mentioned above, with the size distribution and velocities of the ejected fragments, the amount of drilling energy consumed in the kinetic energy of the particles can be estimated. Finally, the fracture energy can be estimated using the sizes of the craters and the fragments size distribution. A shortcoming of this method is that it focuses only on the bitrock interaction processes rather than the entire drilling process. For example, this method does not take into account the energy consumption macroscopic friction of the drill string etc.

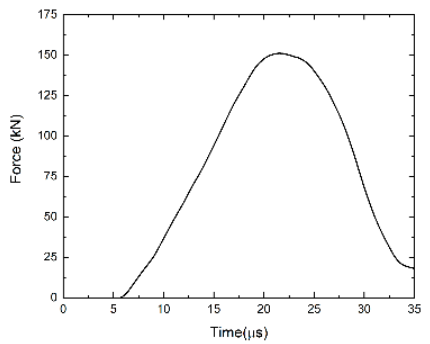

(a)

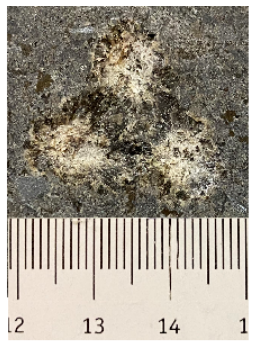

(b)

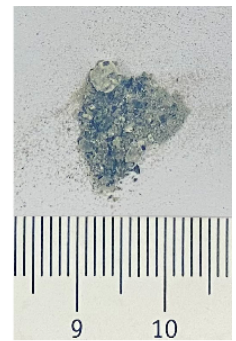

(c)

Fig. 6. (a) Dynamic indentation force as a function of time for a typical test; (b) the craters after the typical test; and (c) the fragments after the typical test.

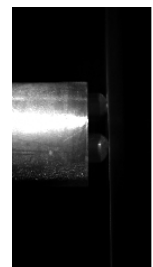

(a)

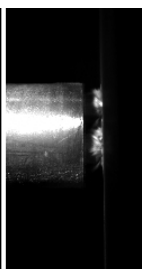

(b)

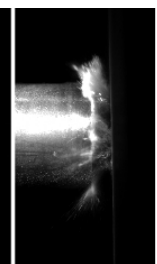

(c)

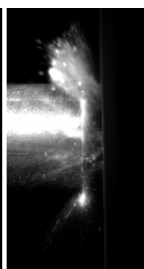

(d)

Fig. 7. The high-speed images for the movement of fragments (the interframe time is $125 \mu \mathrm{s}$ ).

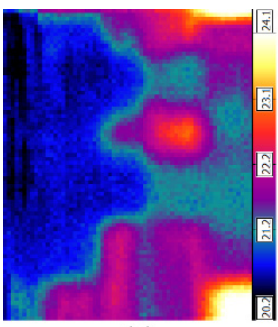

(a)

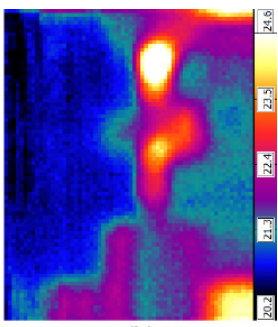

(b)

Fig. 8. The infrared images before and after the impact (the interframe time is $189 \mu$ s).

\section{Conclusions and future work}

In this paper, a numerical model was constructed using the particle flow code (PFC2D). The stress wave propagation in the bar was evaluated indicating that the numerical model can be used to efficiently simulate the dynamic impact process. In addition, the cracks and the fragments can be successfully produced with this simulation. It was verified that the dynamic 
drilling process of granite at high strain rate can be simulated using this model. Furthermore, the experiments for dynamic percussive drilling were conducted using a modified Hopkinson pressure bar system. A high-speed camera and an infrared camera were used to image the failure processes during the dynamic drilling process. The velocity of the fragments was estimated by the high-speed images and the temperature change at the instantaneous impact was observed through infrared images. The preliminary experimental results indicate that this experimental system can be used to estimate fracture energy, thermal energy, and kinetic energy during the dynamic drilling process. In sum, in the numerical model, the crack lengths and the velocities of the fragments can be obtained and thus the details of the energy consumption during the dynamic percussive drilling process can be investigated. Also, the numerical and experimental methodologies can be used to investigate the energy consumption during percussive drilling. More work needs to be conducted with these methods to obtain the energy consumption of the drilling process under different loading and boundary conditions in future.

\section{References}

[1] L. Rybach, Proc. World Geotherm. Cong. 29 1-23 (2010)

[2] J. Wang, S. Hu, Z. Pang, L. He, P. Zhao, C. Zhu, S. Rao, X. Tang, Y. Kong, L. Luo, Sci. Tech. Rev. 3032 (2012)

[3] K. Breede, K. Dzebisashvili, X. Liu, G. Falcone, Geotherm. Ener. 14 (2013)

[4] C. Karpuz, Energy Efficiency of Drilling Operations, in:Kwame Awuah-Offei, Energy Efficiency in the Minerals Industry: Best Practices and Research Directions, Springer International Publishing, Cham, 71-86 (2018)

[5] L.-L. Guo, Y.-B. Zhang, Y.-J. Zhang, Z.-W. Yu, J.-N. Zhang, Renew. Energ. 126 107-125 (2018)

[6] J. Peng, L.N.Y. Wong, C.I. Teh, Z. Li, Rock Mech. Rock Eng. 51 135-154 (2018)

[7] F.E. Dupriest, Comprehensive Drill Rate Management Process To Maximize ROP, in SPE Annual Technical Conference and Exhibition, 3-6 May, San Antonio, Texas, USA (2006)

[8] B. Lundberg, M. Okrouhlik, Int. J. Impact Eng. 32 1573-1583 (2006)

[9] C. Song, J. Chung, J.-S. Cho, Y.-J. Nam, Adv. Mater. Sci. Eng. 2018 1-13 (2018) 